\title{
VARIATION OF ITERATED BROWNIAN MOTION
}

\author{
KRZYSZTOF BuRDZY
}

University of Washington

1. Introduction and main results. Suppose that $X^{1}, X^{2}$ and $Y$ are independent standard Brownian motions starting from 0 and let

$$
X(t)= \begin{cases}X^{1}(t) & \text { if } t \geq 0 \\ X^{2}(-t) & \text { if } t<0\end{cases}
$$

We will consider the process

$$
\{Z(t) \stackrel{\mathrm{df}}{=} X(Y(t)), t \geq 0\}
$$

which we will call "iterated Brownian motion" or simply IBM. It can be proved that $Z$ uniquely determines $X$ and $Y$ (see Burdzy (1992) for a precise statement). A Law of Iterated Logarithm for IBM is also proved in Burdzy (1992).

We consider IBM to be a process of independent interest but there exists an intriguing relationship between this process (strictly speaking its modification) and "squared Laplacian" which was discovered by Funaki (1979). So far, the probabilistic approach to bi-harmonic functions is much less successful than the probabilistic treatment of harmonic functions. Krylov (1960) and Hochberg (1978) attacked the problem using a signed finitely additive measure with infinite variation. Mạdrecki (1992) and Mądrecki and Rybaczuk (1992) have a genuine probabilistic approach but their processes take values in an exotic space. Both models are used to define stochastic integrals for processes with " 4 -th order" scaling properties. Higher order variations of the process play an important role in Mạdrecki and Rybaczuk's construction of the stochastic integral. It is no surprise that the 4 -th variation of their process is a deterministic linear function. The quadratic variation of their process is, in a suitable sense, a Brownian motion. See (3.16) in Hochberg's paper for a result with similar intuitive content.

In this paper, we study higher order variations of IBM with view towards possible applications to the construction of the stochastic integral with respect to IBM. We prove that the 4 -th variation of IBM is a deterministic linear function. This clearly means that the quadratic variation is infinite (although we do not prove this). We show that, in a weak sense, the "signed quadratic variation" of IBM is distributed like Brownian motion.

Suppose that $\Lambda=\left\{s=t_{0} \leq t_{1} \leq \cdots \leq t_{n}=t\right\}$ is a partition of $[s, t]$. The mesh of the partition $\Lambda$ is defined as $|\Lambda| \stackrel{\text { df }}{=} \max _{1 \leq k \leq n}\left|t_{k}-t_{k-1}\right|$.

Supported in part by NSF grant DMS 91-00244 and AMS Centennial Research Fellowship 
Theorem 1. (i) Fix some $0 \leq s<t$. The following limit exists in $L^{p}$ for every $p<\infty$.

$$
\lim _{|\Lambda| \rightarrow 0} \sum_{k=1}^{n}\left(Z\left(t_{k}\right)-Z\left(t_{k-1}\right)\right)^{4}=3(t-s)
$$

(ii) Suppose in addition that $t_{k}-t_{k-1}=(t-s) / n$ for every $k$. Then

$$
\lim _{|\Lambda| \rightarrow 0} \sum_{k=1}^{n}\left(Z\left(t_{k}\right)-Z\left(t_{k-1}\right)\right)^{3}=0 \quad \text { in } L^{p}, p<\infty .
$$

Theorem 2. Suppose that $t_{0}=0$ and $t_{k}-t_{k-1}=1 / n$ for $k \geq 1$. Let

$$
V_{n}\left(t_{m}\right)=\sum_{k=1}^{m}\left(Z\left(t_{k}\right)-Z\left(t_{k-1}\right)\right)^{2} \operatorname{sgn}\left(Z\left(t_{k}\right)-Z\left(t_{k-1}\right)\right) .
$$

Extend $V_{n}$ continuously to $[0, \infty)$ by linear interpolation on each interval $\left[t_{k-1}, t_{k}\right]$. The processes $\left\{V_{n}(s), s \geq 0\right\}$ converge in distribution as $n \rightarrow \infty$ to a Brownian motion $\{B(s), s \geq 0\}$ with variance $\operatorname{Var} B(s)=3 s$.

Remarks. (i) The assumption that $t_{k}-t_{k-1}=t_{j}-t_{j-1}$ for all $j$ and $k$ is imposed for convenience in Theorem 1 (ii) and Theorem 2. The assumption seems to be unnecessary but it makes the calculations somewhat more manageable.

(ii) A heuristic argument suggests that for a fixed $s>0$, the sequence $\left\{V_{n}(s)\right\}_{n \geq 1}$ has no subsequences converging in probability.

(iii) The models considered by Funaki (1979) and Mądrecki and Rybaczuk (1992) involve complex numbers. It might be worth having a look at the complex version of IBM. Suppose that $Y$ is a standard one-dimensional Brownian motion, $X$ is a two-sided complex (i.e., two-dimensional but written in complex notation) Brownian motion and $Z(t)=X(Y(t))$. Let $V_{n}\left(t_{m}\right)=\sum_{k=1}^{m}\left(Z\left(t_{k}\right)-Z\left(t_{k-1}\right)\right)^{2}$, in the notation of Theorem 2. Then Theorem 2 holds for this complex analogue of quadratic variation. The limiting process $B$ for $V_{n}$ 's is a complex (i.e., two-dimensional) Brownian motion with the quadratic variation 3 times as large as the standard one. This result may be proved just like Theorem 2 by using the method of moments.

The proof of Theorem 2 is based on estimates of moments of $V_{n}$ 's. The estimates are quite delicate and it would take enormous amount of space to write them down in all detail. We will carefully examine one crucial estimate and indicate how this can be generalized to other moments.

We would like to thank Ron Pyke for simple proofs of Lemmas 1 and 2 below.

2. Proofs. Throughout the paper, $c$ will stand for a strictly positive and finite constant which may change the value from line to line.

We will need the following standard estimate. Let $a>0$.

$$
\begin{aligned}
\int_{a}^{\infty} x^{2} & \frac{1}{\sqrt{2 \pi t}} \exp \left(-x^{2} / 2 t\right) d x \\
& =-\left.x \sqrt{t / 2 \pi} \exp \left(-x^{2} / 2 t\right)\right|_{x=a} ^{x=\infty}+\int_{a}^{\infty} \sqrt{t / 2 \pi} \exp \left(-x^{2} / 2 t\right) d x \\
& \leq a \sqrt{t / 2 \pi} \exp \left(-a^{2} / 2 t\right)+\int_{a}^{\infty}(x / a) \sqrt{t / 2 \pi} \exp \left(-x^{2} / 2 t\right) d x \\
& =a \sqrt{t / 2 \pi} \exp \left(-a^{2} / 2 t\right)+(t / a) \sqrt{t / 2 \pi} \exp \left(-a^{2} / 2 t\right) \\
& =(a+t / a) \sqrt{t / 2 \pi} \exp \left(-a^{2} / 2 t\right) .
\end{aligned}
$$


The next estimate may be derived in an analogous way using integration by parts.

$$
\int_{a}^{\infty} x^{4} \frac{1}{\sqrt{2 \pi t}} \exp \left(-x^{2} / 2 t\right) d x \leq\left(a^{3}+t a+t^{2} / a\right) \sqrt{t / 2 \pi} \exp \left(-a^{2} / 2 t\right) .
$$

Lemma 1. Suppose that for every (integer) $k \geq 1, k \neq 2$, the $k$-th moment of a random variable $R$ is the same as that of a normal random variable $U$ with mean 0 and variance $\sigma^{2}$. Then $R$ and $U$ have the same distribution.

The point of the lemma is that we do not assume that the variances of $R$ and $U$ are identical. The lemma would follow immediately from known results (see Durrett (1991) Theorem (3.9)) if we added this assumption.

Proof. The $2 k$-th moment $\mu_{2 k}$ of $U$ is equal to $\sigma^{2 k}(2 k-1) !$ !. Thus

$$
\limsup _{k \rightarrow \infty} \mu_{2 k}^{1 / 2 k} / 2 k=\limsup _{k \rightarrow \infty}\left(\sigma^{2 k}(2 k-1) ! !\right)^{1 / 2 k} / 2 k=0 .
$$

Durrett (1991) shows in the proof of Theorem (3.9) that this implies that the characteristic function $\varphi_{U}$ has the following series expansion valid on the whole real line.

$$
\varphi_{U}(t)=1+\sum_{k=1}^{\infty} \frac{t^{k}}{k !} \varphi_{U}^{(k)}(0)
$$

The characteristic function $\varphi_{R}$ of $R$ is represented by an analogous series. For every $k \neq 2$, the $k$-th moment of $R$ is the same as that of $U$ so $\varphi_{R}^{(k)}(0)=\varphi_{U}^{(k)}(0)$ and it follows that the series for $\varphi_{R}$ and $\varphi_{U}$ may differ by at most one term. Hence

$$
\varphi_{R}(t)=\varphi_{U}(t)+a t^{2}
$$

and

$$
\varphi_{R}(t) / t^{2}=\varphi_{U}(t) / t^{2}+a
$$

for all $t \neq 0$. Since $\left|\varphi_{U}(t)\right| \leq 1$ and $\left|\varphi_{R}(t)\right| \leq 1$,

$$
0=\lim _{t \rightarrow \infty} \varphi_{R}(t) / t^{2}=\lim _{t \rightarrow \infty} \varphi_{U}(t) / t^{2}+a=a .
$$

It follows that $a=0$ and, therefore, $U$ and $R$ have identical characteristic functions.

Lemma 2. Let $f_{\sigma}(x)$ denote the centered normal density with standard deviation $\sigma$ and let $\psi(x, \sigma)=f_{\sigma}(0)-f_{\sigma}(x)$. For all $k, n \geq 0$ there exists $c=c(k, n)<\infty$ such that

$$
\int_{-\infty}^{\infty}|x|^{n} \psi\left(x, \rho_{1}\right) \ldots \psi\left(x, \rho_{k}\right) f_{\sigma}(x) d x \leq c \rho_{1}^{-3} \ldots \rho_{k}^{-3} \sigma^{n+2 k}
$$

for all $\rho_{1}, \ldots, \rho_{k} \geq 0$ (c does not depend on $\rho_{j}$ 's or $\sigma$ ).

Proof. Since $1-e^{-y} \leq y$ for all $y \geq 0$,

$$
\psi(x, \rho)=\frac{1}{\sqrt{2 \pi} \rho}\left(1-\exp \left(-x^{2} / 2 \rho^{2}\right)\right) \leq \frac{1}{2 \sqrt{2 \pi}} \frac{x^{2}}{\rho^{3}} .
$$


Let $\xi$ denote a standard normal random variable. Then the integral in the statement of the lemma equals

$$
E|\sigma \xi|^{n} \prod_{j=1}^{k} \psi\left(\sigma \xi, \rho_{j}\right) \leq E|\sigma \xi|^{n+2 k}(2 \sqrt{2 \pi})^{-k} \prod_{j=1}^{k} \rho_{j}^{-3}=c(k, n) \sigma^{n+2 k} \prod_{j=1}^{k} \rho_{j}^{-3}
$$

Proof of Theorem 1 (i). We will only prove the convergence in $L^{p}$ for $p=2$. The general case may be treated in an analogous way.

Recall that $\Lambda=\left\{s=t_{0} \leq t_{1} \leq \cdots \leq t_{n}=t\right\}$ is a partition of $[s, t]$. Let $\Delta_{i} t \stackrel{\text { df }}{=} t_{i}-t_{i-1}$ and $\Delta_{i} Z \stackrel{\text { df }}{=} Z\left(t_{i}\right)-Z\left(t_{i-1}\right)$. We have

$$
\begin{aligned}
{\left[\sum_{i=1}^{n}\left(\Delta_{i} Z\right)^{4}-3(t-s)\right]^{2} } & =\left[\sum_{i=1}^{n}\left(\left(\Delta_{i} Z\right)^{4}-3 \Delta_{i} t\right)\right]^{2} \\
& =\sum_{i, j=1}^{n}\left(\left(\Delta_{i} Z\right)^{4}-3 \Delta_{i} t\right)\left(\left(\Delta_{j} Z\right)^{4}-3 \Delta_{j} t\right) .
\end{aligned}
$$

It will suffice to prove that the expectation of the above random variable goes to 0 as $|\Lambda|$ goes to 0 .

Fix some $\alpha \in(0,1)$ and suppose that $i \neq j$. Fix some numbers $u_{i-1}<u_{i}<u_{j-1}<u_{j}$. We will compute

$$
E\left(\left(\Delta_{i} Z\right)^{4}-3 \Delta_{i} t\right)\left(\left(\Delta_{j} Z\right)^{4}-3 \Delta_{j} t\right)
$$

given

$$
A_{1}=A_{1}\left(u_{i-1}, u_{i}, u_{j-1}, u_{j}\right) \stackrel{\mathrm{df}}{=}\left\{Y\left(t_{i-1}\right)=u_{i-1}, Y\left(t_{i}\right)=u_{i}, Y\left(t_{j-1}\right)=u_{j-1}, Y\left(t_{j}\right)=u_{j}\right\} .
$$

Given this condition, the processes $\left\{X\left(u_{i}+t\right)-X\left(u_{i}\right), t \geq 0\right\}$ and $\left\{X\left(u_{i}-t\right)-X\left(u_{i}\right), t \geq 0\right\}$ are independent standard Brownian motions. Given $A_{1}$, the random variable $\left(\Delta_{i} Z\right)^{4}-3 \Delta_{i} t$ is defined in terms of the first process and $\left(\Delta_{j} Z\right)^{4}-3 \Delta_{j} t$ is defined in terms of the second one. Since $E\left(X\left(s_{1}\right)-X\left(s_{2}\right)\right)^{4}=3\left(s_{1}-s_{2}\right)^{2}$, it follows that

$$
\begin{aligned}
& \begin{aligned}
E\left(\left(\left(\Delta_{i} Z\right)^{4}-\right.\right. & \left.\left.3 \Delta_{i} t\right)\left(\left(\Delta_{j} Z\right)^{4}-3 \Delta_{j} t\right) \mid A_{1}\right) \\
& =E\left(\left(\Delta_{i} Z\right)^{4}-3 \Delta_{i} t \mid A_{1}\right) E\left(\left(\Delta_{j} Z\right)^{4}-3 \Delta_{j} t \mid A_{1}\right) \\
& =E\left(\left(X\left(u_{i}\right)-X\left(u_{i-1}\right)\right)^{4}-3 \Delta_{i} t \mid A_{1}\right) E\left(\left(X\left(u_{j}\right)-X\left(u_{j-1}\right)\right)^{4}-3 \Delta_{j} t \mid A_{1}\right) \\
& =\left(3\left(u_{i}-u_{i-1}\right)^{2}-3 \Delta_{i} t\right)\left(3\left(u_{j}-u_{j-1}\right)^{2}-3 \Delta_{j} t\right) .
\end{aligned}
\end{aligned}
$$

The same argument works for any $u_{i-1}, u_{i}, u_{j-1}$ and $u_{j}$ such that the interval with endpoints $u_{i-1}$ and $u_{i}$ is disjoint from the interval with endpoints $u_{j-1}$ and $u_{j}$.

Suppose that $r>2|\Lambda|^{\alpha / 2}$. Let

$$
\begin{aligned}
A_{2} & =A_{2}(r) \\
& \stackrel{\mathrm{df}}{=}\left\{\left|Y\left(t_{i}\right)-Y\left(t_{j-1}\right)\right|=r,\left|Y\left(t_{i-1}\right)-Y\left(t_{i}\right)\right|<\Delta_{i} t^{\alpha / 2},\left|Y\left(t_{j}\right)-Y\left(t_{j-1}\right)\right|<\Delta_{j} t^{\alpha / 2}\right\} .
\end{aligned}
$$

The increments $Y\left(t_{i-1}\right)-Y\left(t_{i}\right)$ and $Y\left(t_{j}\right)-Y\left(t_{j-1}\right)$ are independent given $A_{2}$. If the event $A_{2}$ occurs then the interval with endpoints $Y\left(t_{i-1}\right)$ and $Y\left(t_{i}\right)$ is disjoint from the interval 
with endpoints $Y\left(t_{j-1}\right)$ and $Y\left(t_{j}\right)$. Hence we may integrate over suitable $u_{i-1}, u_{i}, u_{j-1}$ and $u_{j}$ in (8) to obtain

$$
\begin{aligned}
E\left(\left(\left(\Delta_{i} Z\right)^{4}-\right.\right. & \left.\left.3 \Delta_{i} t\right)\left(\left(\Delta_{j} Z\right)^{4}-3 \Delta_{j} t\right) \mid A_{2}\right) \\
= & \int_{-\Delta_{i} t^{\alpha / 2}}^{\Delta_{i} t^{\alpha / 2}}\left(3 u^{2}-3 \Delta_{i} t\right) \frac{1}{\sqrt{2 \pi \Delta_{i} t}} \exp \left(-u^{2} / 2 \Delta_{i} t\right) d u \times \\
& \times \int_{-\Delta_{j} t^{\alpha / 2}}^{\Delta_{j} t^{\alpha / 2}}\left(3 v^{2}-3 \Delta_{j} t\right) \frac{1}{\sqrt{2 \pi \Delta_{j} t}} \exp \left(-v^{2} / 2 \Delta_{j} t\right) d v .
\end{aligned}
$$

Since

$$
\int_{-\infty}^{\infty}\left(3 u^{2}-3 \Delta_{i} t\right) \frac{1}{\sqrt{2 \pi \Delta_{i} t}} \exp \left(-u^{2} / 2 \Delta_{i} t\right) d u=0,
$$

(5) implies that for some $\beta>0$ and small $\Delta_{i} t$,

$$
\begin{aligned}
\mid \int_{-\Delta_{i} t^{\alpha / 2}}^{\Delta_{i} t^{\alpha / 2}} & \left(3 u^{2}-3 \Delta_{i} t\right) \frac{1}{\sqrt{2 \pi \Delta_{i} t}} \exp \left(-u^{2} / 2 \Delta_{i} t\right) d u \mid \\
& =\left|2 \int_{\Delta_{i} t^{\alpha / 2}}^{\infty}\left(3 u^{2}-3 \Delta_{i} t\right) \frac{1}{\sqrt{2 \pi \Delta_{i} t}} \exp \left(-u^{2} / 2 \Delta_{i} t\right) d u\right| \\
& \leq \int_{\Delta_{i} t^{\alpha / 2}}^{\infty} 6 u^{2} \frac{1}{\sqrt{2 \pi \Delta_{i} t}} \exp \left(-u^{2} / 2 \Delta_{i} t\right) d u \\
& \leq 6\left(\Delta_{i} t^{\alpha / 2}+\Delta_{i} t / \Delta_{i} t^{\alpha / 2}\right) \sqrt{\Delta_{i} t / 2 \pi} \exp \left(-\Delta_{i} t^{\alpha} / 2 \Delta_{i} t\right) \\
& \leq \exp \left(-\Delta_{i} t^{-\beta}\right) .
\end{aligned}
$$

This and (9) show that for small $|\Lambda|$

$$
\left|E\left(\left(\left(\Delta_{i} Z\right)^{4}-3 \Delta_{i} t\right)\left(\left(\Delta_{j} Z\right)^{4}-3 \Delta_{j} t\right) \mid A_{2}\right)\right| \leq \exp \left(-\Delta_{i} t^{-\beta}-\Delta_{j} t^{-\beta}\right) .
$$

Let

$$
\begin{aligned}
& A_{3}=A_{3}(|\Lambda|) \\
& \stackrel{\mathrm{d} f}{=}\left\{\left|Y\left(t_{i}\right)-Y\left(t_{j-1}\right)\right|>2|\Lambda|^{\alpha / 2},\left|Y\left(t_{i-1}\right)-Y\left(t_{i}\right)\right|<\Delta_{i} t^{\alpha / 2},\left|Y\left(t_{j}\right)-Y\left(t_{j-1}\right)\right|<\Delta_{j} t^{\alpha / 2}\right\} .
\end{aligned}
$$

It follows from (11) that

$$
\begin{aligned}
\mid E\left(( ( \Delta _ { i } Z ) ^ { 4 } - 3 \Delta _ { i } t ) \left(\left(\Delta_{j} Z\right)^{4}-\right.\right. & \left.\left.3 \Delta_{j} t\right) \mathbf{1}_{A_{3}}\right) \mid \\
& \leq\left|E\left(\left(\left(\Delta_{i} Z\right)^{4}-3 \Delta_{i} t\right)\left(\left(\Delta_{j} Z\right)^{4}-3 \Delta_{j} t\right) \mid A_{3}\right)\right| \\
& \leq \exp \left(-\Delta_{i} t^{-\beta}-\Delta_{j} t^{-\beta}\right) .
\end{aligned}
$$

Since $E X_{t}^{8}=c t^{4}$,

$$
E\left(\left(\Delta_{i} Z\right)^{8}+9\left(\Delta_{i} t\right)^{2} \mid Y\left(t_{i-1}\right)-Y\left(t_{i}\right)=r\right)=c r^{4}+9\left(\Delta_{i} t\right)^{2} .
$$

An argument similar to that in (10) (except that we would use (6) rather than (5)) gives for small $\Delta_{i} t$ and some $\eta>0$

$$
\begin{aligned}
& \left.\mid E\left(\left(\Delta_{i} Z\right)^{8}+9\left(\Delta_{i} t\right)^{2}\right) \mathbf{1}_{\left\{Y\left(t_{i-1}\right)-Y\left(t_{i}\right)>\Delta_{i}^{\alpha / 2}\right\}}\right) \mid \\
& \quad=\left|2 \int_{\Delta_{i} t^{\alpha / 2}}^{\infty}\left(c u^{4}+9\left(\Delta_{i} t\right)^{2}\right) \frac{1}{\sqrt{2 \pi \Delta_{i} t}} \exp \left(-u^{2} / 2 \Delta_{i} t\right) d u\right| \leq \exp \left(-\Delta_{i} t^{-\eta}\right) .
\end{aligned}
$$


Let

$$
\begin{aligned}
A_{4}=A_{4}(|\Lambda|) \stackrel{\mathrm{df}}{=}\left\{\left|Y\left(t_{i}\right)-Y\left(t_{j-1}\right)\right|>2|\Lambda|^{\alpha / 2}\right\} \cap \\
\\
\cap\left[\left\{\left|Y\left(t_{i-1}\right)-Y\left(t_{i}\right)\right|>\Delta_{i} t^{\alpha / 2}\right\} \cup\left\{\left|Y\left(t_{j}\right)-Y\left(t_{j-1}\right)\right|>\Delta_{j} t^{\alpha / 2}\right\}\right] .
\end{aligned}
$$

Then (14) yields

$$
\begin{aligned}
& \left|E\left(\left(\left(\Delta_{i} Z\right)^{4}-3 \Delta_{i} t\right)\left(\left(\Delta_{j} Z\right)^{4}-3 \Delta_{j} t\right) \mathbf{1}_{A_{4}}\right)\right| \\
& \quad \leq\left(E\left(\left(\Delta_{i} Z\right)^{4}-3 \Delta_{i} t\right)^{2} \mathbf{1}_{A_{4}}\right)^{1 / 2}\left(E\left(\left(\Delta_{j} Z\right)^{4}-3 \Delta_{j} t\right)^{2} \mathbf{1}_{A_{4}}\right)^{1 / 2} \\
& \quad \leq\left(E 2\left(\left(\Delta_{i} Z\right)^{8}+9\left(\Delta_{i} t\right)^{2}\right) \mathbf{1}_{A_{4}}\right)^{1 / 2}\left(E 2\left(\left(\Delta_{j} Z\right)^{8}+9\left(\Delta_{j} t\right)^{2}\right) \mathbf{1}_{A_{4}}\right)^{1 / 2} \\
& \quad \leq 2 \exp \left(-\Delta_{i} t^{-\eta} / 2-\Delta_{j} t^{-\eta} / 2\right) .
\end{aligned}
$$

We define $A_{5}=A_{5}(|\Lambda|)$ to be $\left\{\left|Y\left(t_{i}\right)-Y\left(t_{j-1}\right)\right|>2|\Lambda|^{\alpha / 2}\right\}$. Combining (12) and (15) yields for small $\Delta_{i} t$ and $\Delta_{j} t$

$$
\begin{aligned}
\mid E\left(\left(\left(\Delta_{i} Z\right)^{4}-\right.\right. & \left.\left.3 \Delta_{i} t\right)\left(\left(\Delta_{j} Z\right)^{4}-3 \Delta_{j} t\right) \mathbf{1}_{A_{5}}\right) \mid \\
& \leq \exp \left(-\Delta_{i} t^{-\beta}-\Delta_{j} t^{-\beta}\right)+2 \exp \left(-\Delta_{i} t^{-\eta} / 2-\Delta_{j} t^{-\eta} / 2\right) \\
& \leq 3 \exp \left(-2 \Delta_{i} t^{-\beta}-\Delta_{j} t^{-\eta}\right) .
\end{aligned}
$$

Choose $\alpha, \gamma \in(0,1)$ such that $\delta \stackrel{\mathrm{df}}{=} \alpha / 2-\gamma / 2>0$. Let

$$
A_{6}=\left\{\left|Y\left(t_{i}\right)-Y\left(t_{j-1}\right)\right| \leq 2|\Lambda|^{\alpha / 2}\right\}
$$

and suppose that $\left|t_{i}-t_{j-1}\right|>|\Lambda|^{\gamma}$. Taking the expectation on both sides of (13) gives

$$
E\left(\left(\Delta_{i} Z\right)^{8}+9\left(\Delta_{i} t\right)^{2}\right)=c \Delta_{i} t^{2} .
$$

It is easy to see that

$$
P\left(A_{6}\right) \leq c|\Lambda|^{\alpha / 2} /|\Lambda|^{\gamma / 2}=c|\Lambda|^{\delta} .
$$

By the independence of increments of $Y$,

$$
\begin{aligned}
& E\left(\left(\left(\Delta_{i} Z\right)^{8}+9\left(\Delta_{i} t\right)^{2}\right) \mathbf{1}_{A_{6}}\right)=c \Delta_{i} t^{2} P\left(A_{6}\right)=c \Delta_{i} t^{2}|\Lambda|^{\delta}, \\
& E\left(\left(\left(\Delta_{j} Z\right)^{8}+9\left(\Delta_{j} t\right)^{2}\right) \mathbf{1}_{A_{6}}\right)=c \Delta_{j} t^{2}|\Lambda|^{\delta} .
\end{aligned}
$$

Hence

$$
\begin{aligned}
& \left|E\left(\left(\left(\Delta_{i} Z\right)^{4}-3 \Delta_{i} t\right)\left(\left(\Delta_{j} Z\right)^{4}-3 \Delta_{j} t\right) \mathbf{1}_{A_{6}}\right)\right| \\
& \quad \leq\left(E\left(\left(\Delta_{i} Z\right)^{4}-3 \Delta_{i} t\right)^{2} \mathbf{1}_{A_{6}}\right)^{1 / 2}\left(E\left(\left(\Delta_{j} Z\right)^{4}-3 \Delta_{j} t\right)^{2} \mathbf{1}_{A_{6}}\right)^{1 / 2} \\
& \quad \leq\left(E 2\left(\left(\Delta_{i} Z\right)^{8}+9\left(\Delta_{i} t\right)^{2}\right) \mathbf{1}_{A_{6}}\right)^{1 / 2}\left(E 2\left(\left(\Delta_{j} Z\right)^{8}+9\left(\Delta_{j} t\right)^{2}\right) \mathbf{1}_{A_{6}}\right)^{1 / 2} \\
& \quad \leq 2\left(c \Delta_{i} t^{2}|\Lambda|^{\delta}\right)^{1 / 2}\left(c \Delta_{j} t^{2}|\Lambda|^{\delta}\right)^{1 / 2} \\
& \quad=2 c \Delta_{i} t \Delta_{j} t|\Lambda|^{\delta} .
\end{aligned}
$$

A similar application of the Schwarz inequality gives

$$
\mid E\left(\left(\left(\Delta_{i} Z\right)^{4}-3 \Delta_{i} t\right)\left(\left(\Delta_{j} Z\right)^{4}-3 \Delta_{j} t\right) \mid \leq c \Delta_{i} t \Delta_{j} t\right.
$$


for any $i$ and $j$.

We conclude from (16) and (17) that for sufficiently small $|\Lambda|$

$$
\begin{aligned}
& \sum_{\substack{i, j=1 \\
\left|t_{i}-t_{j-1}\right|>|\Lambda|^{\gamma}}}^{n}\left|E\left(\left(\Delta_{i} Z\right)^{4}-3 \Delta_{i} t\right)\left(\left(\Delta_{j} Z\right)^{4}-3 \Delta_{j} t\right)\right| \\
& \leq \sum_{\substack{i, j=1 \\
\left|t_{i}-t_{j-1}\right|>|\Lambda|^{\gamma}}}^{n}\left(3 \exp \left(-2 \Delta_{i} t^{-\beta}-\Delta_{j} t^{-\eta}\right)+2 c \Delta_{i} t \Delta_{j} t|\Lambda|^{\delta}\right) \\
& \leq c|\Lambda|^{\delta} .
\end{aligned}
$$

As for the remaining terms, we use the estimate (18).

$$
\sum_{\substack{i, j=1 \\\left|t_{i}-t_{j-1}\right| \leq|\Lambda|^{\gamma}}}^{n}\left|E\left(\left(\Delta_{i} Z\right)^{4}-3 \Delta_{i} t\right)\left(\left(\Delta_{j} Z\right)^{4}-3 \Delta_{j} t\right)\right| \leq \sum_{\substack{i, j=1 \\\left|t_{i}-t_{j-1}\right| \leq|\Lambda|^{\gamma}}}^{n} c \Delta_{i} t \Delta_{j} t \leq c|\Lambda|^{\gamma} .
$$

This and (19) show that

$$
\sum_{i, j=1}^{n}\left|E\left(\left(\Delta_{i} Z\right)^{4}-3 \Delta_{i} t\right)\left(\left(\Delta_{j} Z\right)^{4}-3 \Delta_{j} t\right)\right| \rightarrow 0
$$

as $|\Lambda| \rightarrow 0$. This completes the proof of (3) in the case $p=2$. We can prove in a similar way that

$$
\lim _{|\Lambda| \rightarrow 0} \sum_{j_{1}, \ldots, j_{p}=1}^{n}\left|E \prod_{k=1}^{p}\left(\left(\Delta_{j_{k}} Z\right)^{4}-3 \Delta_{j_{k}} t\right)\right|=0
$$

for any $p<\infty$. This can be used to show that the limit in (3) exists in $L^{p}$ for every $p<\infty$.

Proof of Theorem 2. The proof will be based on the method of moments, i.e., we will show that the moments of $V$ converge to the moments of $B$.

Recall that $t_{0}=0$ and $t_{k}-t_{k-1}=1 / n$ for $k \geq 1$. Fix some $0 \leq s_{1}<s_{2}$. Let $\Theta=\Theta(n)$ be the set of all $k$ such that $s_{1} \leq t_{k-1}<t_{k} \leq s_{2}$. The set $\Theta(n)$ is non-empty for sufficiently large $n$. Recall that $\Delta_{k} t=t_{k}-t_{k-1}, \Delta_{k} Z=Z\left(t_{k}\right)-Z\left(t_{k-1}\right), \Delta_{k} Y=Y\left(t_{k}\right)-Y\left(t_{k-1}\right)$ and let

$$
\Delta_{k}^{ \pm} Z^{2}=\left(Z\left(t_{k}\right)-Z\left(t_{k-1}\right)\right)^{2} \operatorname{sgn}\left(Z\left(t_{k}\right)-Z\left(t_{k-1}\right)\right) .
$$

We start with some estimates needed for computing the moments of the increments of $V_{n}$.

For every $s$, the distribution of $X(s)$ is normal so $E X^{2 j}(s)=(2 j-1) ! !|s|^{j}$ (Durrett (1991), Excercise 3.18). By conditioning on the value of $\Delta_{k} Y$ we obtain for some $d_{j}>0$,

$$
\begin{aligned}
E\left(\Delta_{k} Z\right)^{2 j} & =\int_{-\infty}^{\infty} E X^{2 j}(s) \frac{1}{\sqrt{2 \pi \Delta_{k} t}} \exp \left(-s^{2} / 2 \Delta_{k} t\right) d s \\
& =\int_{-\infty}^{\infty}(2 j-1) ! !|s|^{j} \frac{1}{\sqrt{2 \pi \Delta_{k} t}} \exp \left(-s^{2} / 2 \Delta_{k} t\right) d s=d_{j}\left(\Delta_{k} t\right)^{j / 2} .
\end{aligned}
$$


Hence, $E\left(\Delta_{k} Z\right)^{2 j}<\infty$ for all $j<\infty$.

The main contribution in our moment estimates will come from the expectations of the form

$$
E\left(\sum_{k_{1}, \ldots, k_{m} \in \Theta}\left(\Delta_{k_{1}}^{ \pm} Z^{2}\right)^{2}\left(\Delta_{k_{2}}^{ \pm} Z^{2}\right)^{2} \ldots\left(\Delta_{k_{m}}^{ \pm} Z^{2}\right)^{2}\right)
$$

Suppose that $m=2$. We have

$$
\begin{aligned}
\sum_{j, k \in \Theta}\left(\Delta_{j}^{ \pm} Z^{2}\right)^{2}\left(\Delta_{k}^{ \pm} Z^{2}\right)^{2} & =\sum_{j, k \in \Theta} \Delta_{j} Z^{4} \Delta_{k} Z^{4} \\
& =\left[\sum_{j \in \Theta}\left(\left(\Delta_{j} Z\right)^{4}-3 \Delta_{j} t\right)\right]^{2}-\sum_{j, k \in \Theta} 9 \Delta_{j} t \Delta_{k} t+2 \sum_{j, k \in \Theta} 3 \Delta_{j} Z^{4} \Delta_{k} t
\end{aligned}
$$

It is easy to check that $d_{2}=3$ in (22). Thus

$$
E 3 \Delta_{j} Z^{4} \Delta_{k} t=9 \Delta_{j} t \Delta_{k} t
$$

and, therefore,

$$
E \sum_{j, k \in \Theta}\left(\Delta_{j}^{ \pm} Z^{2}\right)^{2}\left(\Delta_{k}^{ \pm} Z^{2}\right)^{2}=E\left[\sum_{j \in \Theta}\left(\left(\Delta_{j} Z\right)^{4}-3 \Delta_{j} t\right)\right]^{2}+\sum_{j, k \in \Theta} 9 \Delta_{j} t \Delta_{k} t .
$$

The expectation on the right hand side goes to 0 as $n$ goes to $\infty$, by (20). Hence

$$
\lim _{n \rightarrow \infty} E \sum_{j, k \in \Theta}\left(\Delta_{j}^{ \pm} Z^{2}\right)^{2}\left(\Delta_{k}^{ \pm} Z^{2}\right)^{2}=\lim _{n \rightarrow \infty} \sum_{j, k \in \Theta} 9 \Delta_{j} t \Delta_{k} t=9\left(s_{2}-s_{1}\right)^{2} .
$$

In order to estimate the expectations in (23) for $m \geq 3$, we use induction. We will treat only the case $m=3$.

$$
\begin{aligned}
\sum_{i, j, k \in \Theta}\left(\Delta_{i}^{ \pm} Z^{2}\right)^{2}\left(\Delta_{j}^{ \pm} Z^{2}\right)^{2}\left(\Delta_{k}^{ \pm} Z^{2}\right)^{2}=\sum_{i, j, k \in \Theta} \Delta_{i} Z^{4} \Delta_{j} Z^{4} \Delta_{k} Z^{4} \\
=\left[\sum_{j \in \Theta}\left(\left(\Delta_{j} Z\right)^{4}-3 \Delta_{j} t\right)\right]^{3}+\sum_{i, j, k \in \Theta} 27 \Delta_{i} t \Delta_{j} t \Delta_{k} t \\
-3 \sum_{i, j, k \in \Theta} 9 \Delta_{i} Z^{4} \Delta_{j} t \Delta_{k} t+3 \sum_{i, j, k \in \Theta} 3 \Delta_{i} Z^{4} \Delta_{j} Z^{4} \Delta_{k} t
\end{aligned}
$$

By (21), (22) and (24),

$$
\begin{gathered}
\lim _{n \rightarrow \infty} E\left[\sum_{j \in \Theta}\left(\left(\Delta_{j} Z\right)^{4}-3 \Delta_{j} t\right)\right]^{3}=0 \\
\lim _{n \rightarrow \infty} E\left[3 \sum_{i, j, k \in \Theta} 9 \Delta_{i} Z^{4} \Delta_{j} t \Delta_{k} t\right]=\lim _{n \rightarrow \infty} E\left[3 \sum_{i, j, k \in \Theta} 27 \Delta_{i} t \Delta_{j} t \Delta_{k} t\right]=81\left(s_{2}-s_{1}\right)^{3}
\end{gathered}
$$


$\lim _{n \rightarrow \infty} E\left[3 \sum_{i, j, k \in \Theta} 3 \Delta_{i} Z^{4} \Delta_{j} Z^{4} \Delta_{k} t\right]=\lim _{n \rightarrow \infty}\left(s_{2}-s_{1}\right) E\left[3 \sum_{i, j \in \Theta} 3 \Delta_{i} Z^{4} \Delta_{j} Z^{4}\right]=81\left(s_{2}-s_{1}\right)^{3}$.

Hence,

$$
\lim _{n \rightarrow \infty} E \sum_{i, j, k \in \Theta}\left(\Delta_{i}^{ \pm} Z^{2}\right)^{2}\left(\Delta_{j}^{ \pm} Z^{2}\right)^{2}\left(\Delta_{k}^{ \pm} Z^{2}\right)^{2}=\lim _{n \rightarrow \infty} E \sum_{i, j, k \in \Theta} 27 \Delta_{i} t \Delta_{j} t \Delta_{k} t=27\left(s_{2}-s_{1}\right)^{3} .
$$

In the same way, using induction, we may prove that

$$
\lim _{n \rightarrow \infty} E\left(\sum_{k_{1}, \ldots, k_{m} \in \Theta}\left(\Delta_{k_{1}}^{ \pm} Z^{2}\right)^{2}\left(\Delta_{k_{2}}^{ \pm} Z^{2}\right)^{2} \ldots\left(\Delta_{k_{m}}^{ \pm} Z^{2}\right)^{2}\right)=3^{m}\left(s_{2}-s_{1}\right)^{m} .
$$

Suppose that $q_{1}, \ldots, q_{m} \geq 1$ are integers and at least one of them is strictly greater than 1. By Hölder's inequality and (22),

$$
\begin{aligned}
E\left[\left(\Delta_{k_{1}}^{ \pm} Z^{2}\right)^{2 q_{1}}\left(\Delta_{k_{2}}^{ \pm} Z^{2}\right)^{2 q_{2}} \ldots\right. & \left.\left(\Delta_{k_{m}}^{ \pm} Z^{2}\right)^{2 q_{m}}\right] \\
& \leq\left(E\left(\Delta_{k_{1}}^{ \pm} Z^{2}\right)^{2 m q_{1}}\right)^{1 / m} \ldots\left(E\left(\Delta_{k_{m}}^{ \pm} Z^{2}\right)^{2 m q_{m}}\right)^{1 / m} \\
& \leq\left(d_{2 m q_{1}}\left(\Delta_{k_{1}} t\right)^{m q_{1}}\right)^{1 / m} \ldots\left(d_{2 m q_{m}}\left(\Delta_{k_{m}} t\right)^{m q_{m}}\right)^{1 / m} \\
& \leq c(1 / n)^{q_{1}+\cdots+q_{m}},
\end{aligned}
$$

and, therefore,

(26)

$\lim _{n \rightarrow \infty} E\left(\sum_{k_{1}, \ldots, k_{m} \in \Theta}\left(\Delta_{k_{1}}^{ \pm} Z^{2}\right)^{2 q_{1}}\left(\Delta_{k_{2}}^{ \pm} Z^{2}\right)^{2 q_{2}} \ldots\left(\Delta_{k_{m}}^{ \pm} Z^{2}\right)^{2 q_{m}}\right) \leq \lim _{n \rightarrow \infty} n^{m} c(1 / n)^{q_{1}+\cdots+q_{m}}=0$.

The absolute value of the difference between

$$
\sum_{k_{1}, \ldots, k_{m} \in \Theta}\left(\Delta_{k_{1}}^{ \pm} Z^{2}\right)^{2}\left(\Delta_{k_{2}}^{ \pm} Z^{2}\right)^{2} \ldots\left(\Delta_{k_{m}}^{ \pm} Z^{2}\right)^{2}
$$

and

$$
\sum_{\substack{k_{1}, \ldots, k_{m} \in \Theta \\ k_{1}, \ldots, k_{m} \text { distinct }}}\left(\Delta_{k_{1}}^{ \pm} Z^{2}\right)^{2}\left(\Delta_{k_{2}}^{ \pm} Z^{2}\right)^{2} \ldots\left(\Delta_{k_{m}}^{ \pm} Z^{2}\right)^{2}
$$

is bounded by a finite sum of the expressions of the form

$$
\sum_{k_{1}, \ldots, k_{i} \in \Theta}\left(\Delta_{k_{1}}^{ \pm} Z^{2}\right)^{2 q_{1}}\left(\Delta_{k_{2}}^{ \pm} Z^{2}\right)^{2 q_{2}} \ldots\left(\Delta_{k_{i}}^{ \pm} Z^{2}\right)^{2 q_{i}}
$$

where at least one of the $q_{j}$ 's is greater than 1 . It follows from (25) and (26) that

$$
\lim _{n \rightarrow \infty} E\left(\sum_{\substack{k_{1}, \ldots, k_{m} \in \Theta \\ k_{1}, \ldots, k_{m} \text { distinct }}}\left(\Delta_{k_{1}}^{ \pm} Z^{2}\right)^{2}\left(\Delta_{k_{2}}^{ \pm} Z^{2}\right)^{2} \ldots\left(\Delta_{k_{m}}^{ \pm} Z^{2}\right)^{2}\right)=3^{m}\left(s_{2}-s_{1}\right)^{m} .
$$

Next we tackle the expectations of the form

$$
E\left(\sum_{k_{1}, \ldots, k_{m} \in \Theta}\left(\Delta_{k_{1}}^{ \pm} Z^{2}\right)^{q_{1}}\left(\Delta_{k_{2}}^{ \pm} Z^{2}\right)^{q_{2}} \ldots\left(\Delta_{k_{m}}^{ \pm} Z^{2}\right)^{q_{m}}\right)
$$


where $q_{1}, \ldots, q_{m} \geq 1$ are integers but they are not necessarily even. We will tacitly assume that the sum is taken over indices which are pairwise distinct (the other terms appear in sums with different exponents $q_{k}$ ). We will illustrate the method by analyzing in detail only one sum, namely,

$$
E\left(\sum_{\substack{k_{1}, \ldots, k_{4} \in \Theta \\ k_{1}<k_{2}<k_{3}<k_{4}}}\left(\Delta_{k_{1}}^{ \pm} Z^{2}\right)^{2} \Delta_{k_{2}}^{ \pm} Z^{2}\left(\Delta_{k_{3}}^{ \pm} Z^{2}\right)^{2} \Delta_{k_{4}}^{ \pm} Z^{2}\right) .
$$

Note that the indices in the last sum are ordered - the sum with unordered indices may be obtained by adding a finite number of sums with ordered indices. Let

$$
\begin{gathered}
A_{1}=A_{1}\left(u_{k_{1}-1}, u_{k_{1}}, u_{k_{2}-1}, u_{k_{2}}, u_{k_{3}-1}, u_{k_{3}}, u_{k_{4}-1}, u_{k_{4}}\right) \\
\stackrel{\text { df }}{=}\left\{Y\left(t_{k_{i}-1}\right)=u_{k_{i}-1}, Y\left(t_{k_{i}}\right)=u_{k_{i}}, i=1,2,3,4\right\} .
\end{gathered}
$$

Let $A_{2}$ denote the event that there exists a number $a$ such that for each $i$, the interval with endpoints $u_{k_{i}-1}$ and $u_{k_{i}}$ is either contained in $(a, \infty)$ or in $(-\infty, a)$ and that each half-line contains at least one of these intervals. Suppose for a moment that $u_{k_{i}-1}$ and $u_{k_{i}}$ are such that $A_{2}$ holds, for example, the intervals corresponding to $i=1,2$ are in $(a, \infty)$ and the other two are contained in the other half-line. The same argument that leads to (8) gives in the present case

$$
\begin{aligned}
E\left(\left(\Delta_{k_{1}}^{ \pm} Z^{2}\right)^{2} \Delta_{k_{2}}^{ \pm} Z^{2}\left(\Delta_{k_{3}}^{ \pm} Z^{2}\right)^{2} \Delta_{k_{4}}^{ \pm} Z^{2} \mid A_{1}\right) & \\
& =E\left(\left(\Delta_{k_{1}}^{ \pm} Z^{2}\right)^{2} \Delta_{k_{2}}^{ \pm} Z^{2} \mid A_{1}\right) E\left(\left(\Delta_{k_{3}}^{ \pm} Z^{2}\right)^{2} \Delta_{k_{4}}^{ \pm} Z^{2} \mid A_{1}\right) .
\end{aligned}
$$

Both conditional expectations on the right hand side are equal to zero since the random variables have symmetric (conditional) distributions. If the event $A_{2}$ is realized in some other way, the conditional expectation on the left hand side of (28) may be factored in some other way such that at least one conditional expectation on the right hand side is equal to 0 because of symmetry of the involved distribution. Hence,

$$
E\left(\left(\Delta_{k_{1}}^{ \pm} Z^{2}\right)^{2} \Delta_{k_{2}}^{ \pm} Z^{2}\left(\Delta_{k_{3}}^{ \pm} Z^{2}\right)^{2} \Delta_{k_{4}}^{ \pm} Z^{2} \mathbf{1}_{A_{2}}\right)=0 .
$$

For arbitrary values of $u_{i}$ 's,

$$
\begin{aligned}
E\left(\left(\Delta_{k_{1}}^{ \pm} Z^{2}\right)^{2} \Delta_{k_{2}}^{ \pm} Z^{2}\left(\Delta_{k_{3}}^{ \pm} Z^{2}\right)^{2} \Delta_{k_{4}}^{ \pm} Z^{2} \mid A_{1}\right) & \\
\leq & \left(E\left(\left(\Delta_{k_{1}}^{ \pm} Z^{2}\right)^{4} \mid A_{1}\right)\right)^{1 / 2}\left(E\left(\left(\Delta_{k_{2}}^{ \pm} Z^{2}\right)^{2} \mid A_{1}\right)\right)^{1 / 2} \times \\
& \quad \times\left(E\left(\left(\Delta_{k_{3}}^{ \pm} Z^{2}\right)^{4} \mid A_{1}\right)\right)^{1 / 2}\left(E\left(\left(\Delta_{k_{4}}^{ \pm} Z^{2}\right)^{2} \mid A_{1}\right)\right)^{1 / 2} \\
= & \left(E X^{8}\left(\left|u_{k_{1}-1}-u_{k_{1}}\right|\right)\right)^{1 / 2}\left(E X^{4}\left(\left|u_{k_{2}-1}-u_{k_{2}}\right|\right)\right)^{1 / 2} \times \\
& \quad \times\left(E X^{8}\left(\left|u_{k_{3}-1}-u_{k_{3}}\right|\right)\right)^{1 / 2}\left(E X^{4}\left(\left|u_{k_{4}-1}-u_{k_{4}}\right|\right)\right)^{1 / 2} \\
\leq & c\left|u_{k_{1}-1}-u_{k_{1}}\right|^{2}\left|u_{k_{2}-1}-u_{k_{2}}\right|\left|u_{k_{3}-1}-u_{k_{3}}\right|^{2}\left|u_{k_{4}-1}-u_{k_{4}}\right| .
\end{aligned}
$$

Note that

$E\left(\left(\Delta_{k_{1}}^{ \pm} Z^{2}\right)^{2} \Delta_{k_{2}}^{ \pm} Z^{2}\left(\Delta_{k_{3}}^{ \pm} Z^{2}\right)^{2} \Delta_{k_{4}}^{ \pm} Z^{2} \mid A_{1}\left(u_{k_{1}-1}, u_{k_{1}}, u_{k_{2}-1}, u_{k_{2}}, u_{k_{3}-1}, u_{k_{3}}, u_{k_{4}-1}, u_{k_{4}}\right)\right)$

$$
=-E\left(\left(\Delta_{k_{1}}^{ \pm} Z^{2}\right)^{2} \Delta_{k_{2}}^{ \pm} Z^{2}\left(\Delta_{k_{3}}^{ \pm} Z^{2}\right)^{2} \Delta_{k_{4}}^{ \pm} Z^{2} \mid A_{1}\left(u_{k_{1}-1}, u_{k_{1}}, u_{k_{2}}, u_{k_{2}-1}, u_{k_{3}-1}, u_{k_{3}}, u_{k_{4}-1}, u_{k_{4}}\right)\right)
$$


because when we exchange the roles of $u_{k_{2}-1}$ and $u_{k_{2}}$, we change, in a sense, the sign of $\Delta_{k_{2}}^{ \pm} Z^{2}$. For the same reason we have

$E\left(\left(\Delta_{k_{1}}^{ \pm} Z^{2}\right)^{2} \Delta_{k_{2}}^{ \pm} Z^{2}\left(\Delta_{k_{3}}^{ \pm} Z^{2}\right)^{2} \Delta_{k_{4}}^{ \pm} Z^{2} \mid A_{1}\left(u_{k_{1}-1}, u_{k_{1}}, u_{k_{2}-1}, u_{k_{2}}, u_{k_{3}-1}, u_{k_{3}}, u_{k_{4}-1}, u_{k_{4}}\right)\right)$

(32)

$=-E\left(\left(\Delta_{k_{1}}^{ \pm} Z^{2}\right)^{2} \Delta_{k_{2}}^{ \pm} Z^{2}\left(\Delta_{k_{3}}^{ \pm} Z^{2}\right)^{2} \Delta_{k_{4}}^{ \pm} Z^{2} \mid A_{1}\left(u_{k_{1}-1}, u_{k_{1}}, u_{k_{2}-1}, u_{k_{2}}, u_{k_{3}-1}, u_{k_{3}}, u_{k_{4}}, u_{k_{4}-1}\right)\right)$.

Let $\rho=\rho\left(r_{1}, r_{2}, r_{3}, r_{4}\right)=\left|r_{1}\right|+\left|r_{2}\right|+\left|r_{3}\right|+\left|r_{4}\right|$. We claim that

$$
\begin{aligned}
& \left|E\left(\left(\Delta_{k_{1}}^{ \pm} Z^{2}\right)^{2} \Delta_{k_{2}}^{ \pm} Z^{2}\left(\Delta_{k_{3}}^{ \pm} Z^{2}\right)^{2} \Delta_{k_{4}}^{ \pm} Z^{2}\right)\right| \\
& \leq \mid \int\left[\left|r_{1}\right|^{2}\left|r_{2}\right|\left|r_{3}\right|^{2}\left|r_{4}\right| P\left(Y\left(t_{k_{1}}\right)-Y\left(t_{k_{1}-1}\right) \in d r_{1}\right) P\left(Y\left(t_{k_{2}}\right)-Y\left(t_{k_{2}-1}\right) \in d r_{2}\right)\right. \\
& \left.P\left(Y\left(t_{k_{3}}\right)-Y\left(t_{k_{3}-1}\right) \in d r_{3}\right) P\left(Y\left(t_{k_{4}}\right)-Y\left(t_{k_{4}-1}\right) \in d r_{4}\right)\right] \\
& \mathbf{1}_{\left|r_{5}\right| \leq \rho} \mathbf{1}_{\left|r_{6}\right| \leq \rho}\left[P\left(Y\left(t_{k_{2}-1}\right)-Y\left(t_{k_{1}}\right) \in d r_{5}\right) P\left(Y\left(t_{k_{3}-1}\right)-Y\left(t_{k_{2}}\right) \in d r_{6}\right)\right. \\
& \left.\quad-P\left(Y\left(t_{k_{2}-1}\right)-Y\left(t_{k_{1}}\right) \in d\left(r_{5}+r_{2}\right)\right) P\left(Y\left(t_{k_{3}-1}\right)-Y\left(t_{k_{2}}\right) \in d\left(r_{6}-r_{2}\right)\right)\right] \\
& \left.\mathbf{1}_{\left|r_{7}\right| \leq \rho}\left[P\left(Y\left(t_{k_{4}-1}\right)-Y\left(t_{k_{3}}\right) \in d r_{7}\right)-P\left(Y\left(t_{k_{4}-1}\right)-Y\left(t_{k_{3}}\right) \in d\left(r_{7}+r_{4}\right)\right)\right]\right] .
\end{aligned}
$$

The terms in the first pair of large square brackets are justified by (30). The terms in the second and third pair of large square brackets come from (31)-(32). The presence of indicator functions follows from (29).

We will now estimate

$$
\begin{aligned}
& \mid \int \mathbf{1}_{\left|r_{5}\right| \leq \rho} \mathbf{1}_{\left|r_{6}\right| \leq \rho}\left[P\left(Y\left(t_{k_{2}-1}\right)-Y\left(t_{k_{1}}\right) \in d r_{5}\right) P\left(Y\left(t_{k_{3}-1}\right)-Y\left(t_{k_{2}}\right) \in d r_{6}\right)\right. \\
& \left.\quad-P\left(Y\left(t_{k_{2}-1}\right)-Y\left(t_{k_{1}}\right) \in d\left(r_{5}+r_{2}\right)\right) P\left(Y\left(t_{k_{3}-1}\right)-Y\left(t_{k_{2}}\right) \in d\left(r_{6}-r_{2}\right)\right)\right] \mid .
\end{aligned}
$$

Recall $\psi(x, \sigma)$ from Lemma 2. Since $t_{k_{2}-1}-t_{k_{1}}=\left(k_{2}-1-k_{1}\right) / n$, the standard deviation of $Y\left(t_{k_{2}-1}\right)-Y\left(t_{k_{1}}\right)$ is equal to $\left(\left(k_{2}-1-k_{1}\right) / n\right)^{1 / 2}$. Hence

$$
\begin{aligned}
& \left|P\left(Y\left(t_{k_{2}-1}\right)-Y\left(t_{k_{1}}\right) \in d r_{5}\right)-P\left(Y\left(t_{k_{2}-1}\right)-Y\left(t_{k_{1}}\right) \in d\left(r_{5}+r_{2}\right)\right)\right| \\
& \quad \leq\left(\psi\left(r_{5},\left(\left(k_{2}-1-k_{1}\right) / n\right)^{1 / 2}\right)+\psi\left(r_{5}+r_{2},\left(\left(k_{2}-1-k_{1}\right) / n\right)^{1 / 2}\right)\right) d r_{5} \\
& \quad \leq 2 \psi\left(2 \rho,\left(\left(k_{2}-1-k_{1}\right) / n\right)^{1 / 2}\right) d r_{5}
\end{aligned}
$$

provided $\left|r_{5}\right| \leq \rho$. Similarly,

$$
\begin{aligned}
& \left|P\left(Y\left(t_{k_{3}-1}\right)-Y\left(t_{k_{2}}\right) \in d r_{6}\right)-P\left(Y\left(t_{k_{3}-1}\right)-Y\left(t_{k_{2}}\right) \in d\left(r_{6}-r_{2}\right)\right)\right| \\
& \quad \leq 2 \psi\left(2 \rho,\left(\left(k_{3}-1-k_{2}\right) / n\right)^{1 / 2}\right) d r_{6}
\end{aligned}
$$

assuming $\left|r_{6}\right| \leq \rho$. We will assume temporarily that $k_{j}-k_{j-1}>1$ for all $j$ in order to avoid normal variables with zero variance and in order to be able to use Lemma 2 . We will get rid of this assumption later. 
For any reals $a, \Delta a, b$ and $\Delta b$,

$$
|a b-(a+\Delta a)(b+\Delta b)| \leq|a \Delta b|+|b \Delta a|+|\Delta a \Delta b|
$$

This, (35) and (36) imply

$$
\begin{aligned}
& \mid P\left(Y\left(t_{k_{2}-1}\right)-Y\left(t_{k_{1}}\right) \in d r_{5}\right) P\left(Y\left(t_{k_{3}-1}\right)-Y\left(t_{k_{2}}\right) \in d r_{6}\right) \\
& \quad-P\left(Y\left(t_{k_{2}-1}\right)-Y\left(t_{k_{1}}\right) \in d\left(r_{5}+r_{2}\right)\right) P\left(Y\left(t_{k_{3}-1}\right)-Y\left(t_{k_{2}}\right) \in d\left(r_{6}-r_{2}\right)\right) \mid \\
& \leq 2 \psi\left(2 \rho,\left(\left(k_{2}-1-k_{1}\right) / n\right)^{1 / 2}\right) d r_{5} P\left(Y\left(t_{k_{3}-1}\right)-Y\left(t_{k_{2}}\right) \in d r_{6}\right) \\
& \quad+2 \psi\left(2 \rho,\left(\left(k_{3}-1-k_{2}\right) / n\right)^{1 / 2}\right) d r_{6} P\left(Y\left(t_{k_{2}-1}\right)-Y\left(t_{k_{1}}\right) \in d r_{5}\right) \\
& \quad+2 \psi\left(2 \rho,\left(\left(k_{2}-1-k_{1}\right) / n\right)^{1 / 2}\right) d r_{5} 2 \psi\left(2 \rho,\left(\left(k_{3}-1-k_{2}\right) / n\right)^{1 / 2}\right) d r_{6} .
\end{aligned}
$$

The integral in (34) is, therefore, bounded by

$$
\begin{aligned}
& \mid \int \mathbf{1}_{\left|r_{5}\right| \leq \rho} \mathbf{1}_{\left|r_{6}\right| \leq \rho} \\
& \quad\left[2 \psi\left(2 \rho,\left(\left(k_{2}-1-k_{1}\right) / n\right)^{1 / 2}\right) d r_{5} P\left(Y\left(t_{k_{3}-1}\right)-Y\left(t_{k_{2}}\right) \in d r_{6}\right)\right. \\
& \quad+2 \psi\left(2 \rho,\left(\left(k_{3}-1-k_{2}\right) / n\right)^{1 / 2}\right) d r_{6} P\left(Y\left(t_{k_{2}-1}\right)-Y\left(t_{k_{1}}\right) \in d r_{5}\right) \\
& \left.\quad+2 \psi\left(2 \rho,\left(\left(k_{2}-1-k_{1}\right) / n\right)^{1 / 2}\right) d r_{5} 2 \psi\left(2 \rho,\left(\left(k_{3}-1-k_{2}\right) / n\right)^{1 / 2}\right) d r_{6}\right] \mid \\
& \quad \leq c \psi\left(2 \rho,\left(\left(k_{2}-1-k_{1}\right) / n\right)^{1 / 2}\right) \rho^{2}\left(\left(k_{3}-1-k_{2}\right) / n\right)^{-1 / 2} \\
& \quad+c \psi\left(2 \rho,\left(\left(k_{3}-1-k_{2}\right) / n\right)^{1 / 2}\right) \rho^{2}\left(\left(k_{2}-1-k_{1}\right) / n\right)^{-1 / 2} \\
& \quad+c \psi\left(2 \rho,\left(\left(k_{2}-1-k_{1}\right) / n\right)^{1 / 2}\right) \rho^{2} \psi\left(2 \rho,\left(\left(k_{3}-1-k_{2}\right) / n\right)^{1 / 2}\right) .
\end{aligned}
$$

We can prove in the same way that

$$
\begin{aligned}
& \left|\int \mathbf{1}_{\left|r_{7}\right| \leq \rho}\left[P\left(Y\left(t_{k_{4}-1}\right)-Y\left(t_{k_{3}}\right) \in d r_{7}\right)-P\left(Y\left(t_{k_{4}-1}\right)-Y\left(t_{k_{3}}\right) \in d\left(r_{7}+r_{4}\right)\right)\right]\right| \\
& \quad \leq c \psi\left(2 \rho,\left(\left(k_{4}-1-k_{3}\right) / n\right)^{1 / 2}\right) \rho .
\end{aligned}
$$

We now substitute this estimate and (37) into (33) to obtain

$$
\begin{aligned}
& \left|E\left(\left(\Delta_{k_{1}}^{ \pm} Z^{2}\right)^{2} \Delta_{k_{2}}^{ \pm} Z^{2}\left(\Delta_{k_{3}}^{ \pm} Z^{2}\right)^{2} \Delta_{k_{4}}^{ \pm} Z^{2}\right)\right| \\
& \leq\left.\left|\int\right| r_{1}\right|^{2}\left|r_{2}\right|\left|r_{3}\right|^{2}\left|r_{4}\right| P\left(Y\left(t_{k_{1}}\right)-Y\left(t_{k_{1}-1}\right) \in d r_{1}\right) P\left(Y\left(t_{k_{2}}\right)-Y\left(t_{k_{2}-1}\right) \in d r_{2}\right) \\
& P\left(Y\left(t_{k_{3}}\right)-Y\left(t_{k_{3}-1}\right) \in d r_{3}\right) P\left(Y\left(t_{k_{4}}\right)-Y\left(t_{k_{4}-1}\right) \in d r_{4}\right) \\
& {\left[c \psi\left(2 \rho,\left(\left(k_{2}-1-k_{1}\right) / n\right)^{1 / 2}\right) \rho^{2}\left(\left(k_{3}-1-k_{2}\right) / n\right)^{-1 / 2}\right.} \\
& \quad+c \psi\left(2 \rho,\left(\left(k_{3}-1-k_{2}\right) / n\right)^{1 / 2}\right) \rho^{2}\left(\left(k_{2}-1-k_{1}\right) / n\right)^{-1 / 2} \\
& \left.\quad+c \psi\left(2 \rho,\left(\left(k_{2}-1-k_{1}\right) / n\right)^{1 / 2}\right) \rho^{2} \psi\left(2 \rho,\left(\left(k_{3}-1-k_{2}\right) / n\right)^{1 / 2}\right)\right] \\
& c \psi\left(2 \rho,\left(\left(k_{4}-1-k_{3}\right) / n\right)^{1 / 2}\right) \rho \mid
\end{aligned}
$$


By multiplying out the expression in brackets on the right hand side we obtain three terms under the integral sign. The first one is equal to

$$
\begin{gathered}
\int\left|r_{1}\right|^{2}\left|r_{2}\right|\left|r_{3}\right|^{2}\left|r_{4}\right| c \psi\left(2 \rho,\left(\left(k_{2}-1-k_{1}\right) / n\right)^{1 / 2}\right) \rho^{2}\left(\left(k_{3}-1-k_{2}\right) / n\right)^{-1 / 2} \\
c \psi\left(2 \rho,\left(\left(k_{4}-1-k_{3}\right) / n\right)^{1 / 2}\right) \rho P\left(Y\left(t_{k_{1}}\right)-Y\left(t_{k_{1}-1}\right) \in d r_{1}\right) P\left(Y\left(t_{k_{2}}\right)-Y\left(t_{k_{2}-1}\right) \in d r_{2}\right) \\
P\left(Y\left(t_{k_{3}}\right)-Y\left(t_{k_{3}-1}\right) \in d r_{3}\right) P\left(Y\left(t_{k_{4}}\right)-Y\left(t_{k_{4}-1}\right) \in d r_{4}\right) \\
=\int\left|r_{1}\right|^{2}\left|r_{2}\right|\left|r_{3}\right|^{2}\left|r_{4}\right| c \psi\left(2\left(\left|r_{1}\right|+\left|r_{2}\right|+\left|r_{3}\right|+\left|r_{4}\right|\right),\left(\left(k_{2}-1-k_{1}\right) / n\right)^{1 / 2}\right) \\
\left(\left|r_{1}\right|+\left|r_{2}\right|+\left|r_{3}\right|+\left|r_{4}\right|\right)^{2}\left(\left(k_{3}-1-k_{2}\right) / n\right)^{-1 / 2} \\
c \psi\left(2\left(\left|r_{1}\right|+\left|r_{2}\right|+\left|r_{3}\right|+\left|r_{4}\right|\right),\left(\left(k_{4}-1-k_{3}\right) / n\right)^{1 / 2}\right)\left(\left|r_{1}\right|+\left|r_{2}\right|+\left|r_{3}\right|+\left|r_{4}\right|\right) \\
P\left(Y\left(t_{k_{1}}\right)-Y\left(t_{k_{1}-1}\right) \in d r_{1}\right) P\left(Y\left(t_{k_{2}}\right)-Y\left(t_{k_{2}-1}\right) \in d r_{2}\right) \\
\\
P\left(Y\left(t_{k_{3}}\right)-Y\left(t_{k_{3}-1}\right) \in d r_{3}\right) P\left(Y\left(t_{k_{4}}\right)-Y\left(t_{k_{4}-1}\right) \in d r_{4}\right) .
\end{gathered}
$$

Note that

$$
\psi\left(2\left(\left|r_{1}\right|+\left|r_{2}\right|+\left|r_{3}\right|+\left|r_{4}\right|\right),\left(\left(k_{2}-1-k_{1}\right) / n\right)^{1 / 2}\right) \leq \sum_{j=1}^{4} \psi\left(8\left|r_{j}\right|,\left(\left(k_{2}-1-k_{1}\right) / n\right)^{1 / 2}\right)
$$

and the standard deviation of $Y\left(t_{k_{j}}\right)-Y\left(t_{k_{j}-1}\right)$ is equal to $n^{-1 / 2}$. This and Lemma 2 can be used to show that (40) is bounded by

$$
c\left(\left(k_{2}-1-k_{1}\right) / n\right)^{-3 / 2}\left(\left(k_{3}-1-k_{2}\right) / n\right)^{-1 / 2}\left(\left(k_{4}-1-k_{3}\right) / n\right)^{-3 / 2} n^{-13 / 2} .
$$

The other terms in (39) may be treated in a similar way so

$$
\begin{aligned}
& \left|E\left(\left(\Delta_{k_{1}}^{ \pm} Z^{2}\right)^{2} \Delta_{k_{2}}^{ \pm} Z^{2}\left(\Delta_{k_{3}}^{ \pm} Z^{2}\right)^{2} \Delta_{k_{4}}^{ \pm} Z^{2}\right)\right| \\
& \quad \leq c\left(\left(k_{2}-1-k_{1}\right) / n\right)^{-3 / 2}\left(\left(k_{3}-1-k_{2}\right) / n\right)^{-1 / 2}\left(\left(k_{4}-1-k_{3}\right) / n\right)^{-3 / 2} n^{-13 / 2} \\
& \quad+c\left(\left(k_{3}-1-k_{2}\right) / n\right)^{-3 / 2}\left(\left(k_{2}-1-k_{1}\right) / n\right)^{-1 / 2}\left(\left(k_{4}-1-k_{3}\right) / n\right)^{-3 / 2} n^{-13 / 2} \\
& \quad+c\left(\left(k_{2}-1-k_{1}\right) / n\right)^{-3 / 2}\left(\left(k_{3}-1-k_{2}\right) / n\right)^{-3 / 2}\left(\left(k_{4}-1-k_{3}\right) / n\right)^{-3 / 2} n^{-15 / 2} .
\end{aligned}
$$

Now we discuss our temporary assumption that $k_{j}-k_{j-1}>1$. If $k_{4}-k_{3}=1$ then the last term in large square brackets in (33) should be replaced by 1 . If $k_{4}-k_{3}=2$ then the effect of the same term on our estimate is that of a multiplicative constant (see, e.g., (38)). It follows that the terms corresponding to $k_{j}-k_{j-1}=1$ contribute to our sums as much as those corresponding to $k_{j}-k_{j-1}=2$ (up to a multiplicative constant). Having this in 
mind, we may write

$$
\begin{aligned}
& \left|E\left(\sum_{\substack{k_{1}, \ldots, k_{4} \in \Theta \\
k_{1}<k_{2}<k_{3}<k_{4}}}\left(\Delta_{k_{1}}^{ \pm} Z^{2}\right)^{2} \Delta_{k_{2}}^{ \pm} Z^{2}\left(\Delta_{k_{3}}^{ \pm} Z^{2}\right)^{2} \Delta_{k_{4}}^{ \pm} Z^{2}\right)\right| \\
& \quad \leq \sum_{\substack{k_{1}, \ldots, k_{4} \in \Theta \\
k_{1}<k_{2}<k_{3}<k_{4} \\
k_{j}-k_{j}-1>1}}^{\left[c\left(\left(k_{2}-1-k_{1}\right) / n\right)^{-3 / 2}\left(\left(k_{3}-1-k_{2}\right) / n\right)^{-1 / 2}\left(\left(k_{4}-1-k_{3}\right) / n\right)^{-3 / 2} n^{-13 / 2}\right.} \\
& \quad+c\left(\left(k_{3}-1-k_{2}\right) / n\right)^{-3 / 2}\left(\left(k_{2}-1-k_{1}\right) / n\right)^{-1 / 2}\left(\left(k_{4}-1-k_{3}\right) / n\right)^{-3 / 2} n^{-13 / 2} \\
& \left.\quad+c\left(\left(k_{2}-1-k_{1}\right) / n\right)^{-3 / 2}\left(\left(k_{3}-1-k_{2}\right) / n\right)^{-3 / 2}\left(\left(k_{4}-1-k_{3}\right) / n\right)^{-3 / 2} n^{-15 / 2}\right] .
\end{aligned}
$$

We have

$$
\begin{aligned}
& \sum_{\substack{k_{1}, \ldots, k_{4} \in \Theta \\
k_{1}<k_{2}<k_{3}<k_{4} \\
k_{j}-k_{j-1}>1}} c\left(\left(k_{2}-1-k_{1}\right) / n\right)^{-3 / 2}\left(\left(k_{3}-1-k_{2}\right) / n\right)^{-1 / 2}\left(\left(k_{4}-1-k_{3}\right) / n\right)^{-3 / 2} n^{-13 / 2} \\
& \quad \leq c n^{-3} \sum_{k_{1}=1}^{n} \sum_{j_{1}=1}^{\infty} j_{1}^{-3 / 2} \sum_{j_{2}=1}^{n} j_{2}^{-1 / 2} \sum_{j_{3}=1}^{\infty} j_{3}^{-3 / 2} \\
& \quad \leq c n^{-3} n^{1 / 2}=c n^{-3 / 2} .
\end{aligned}
$$

Similar bounds hold for other terms in (42) so that

$$
\left|E\left(\sum_{\substack{k_{1}, \ldots, k_{4} \in \Theta \\ k_{1}<k_{2}<k_{3}<k_{4}}}\left(\Delta_{k_{1}}^{ \pm} Z^{2}\right)^{2} \Delta_{k_{2}}^{ \pm} Z^{2}\left(\Delta_{k_{3}}^{ \pm} Z^{2}\right)^{2} \Delta_{k_{4}}^{ \pm} Z^{2}\right)\right| \leq c n^{-3 / 2}+c n^{-3 / 2}+c n^{-2}
$$

and, therefore,

$$
\lim _{n \rightarrow \infty}\left|E\left(\sum_{\substack{k_{1}, \ldots, k_{4} \in \Theta \\ k_{1}<k_{2}<k_{3}<k_{4}}}\left(\Delta_{k_{1}}^{ \pm} Z^{2}\right)^{2} \Delta_{k_{2}}^{ \pm} Z^{2}\left(\Delta_{k_{3}}^{ \pm} Z^{2}\right)^{2} \Delta_{k_{4}}^{ \pm} Z^{2}\right)\right|=0
$$

Now we will explain how this result may be generalized. Suppose that $q_{j}$ is either equal to 1 or 2 for $j=1, \ldots, m$. The expectation

$$
\left|E\left(\left(\Delta_{k_{1}}^{ \pm} Z^{2}\right)^{q_{1}}\left(\Delta_{k_{2}}^{ \pm} Z^{2}\right)^{q_{2}} \ldots\left(\Delta_{k_{m}}^{ \pm} Z^{2}\right)^{q_{m}}\right)\right|
$$

may be bounded as in (41) by a product of factors corresponding to $\left(\Delta_{k_{j}}^{ \pm} Z^{2}\right)^{q_{j}}$. If $q_{j}=1$ and $j>1$ then the factor is of the form

$$
c\left(\left(k_{j}-1-k_{j-1}\right) / n\right)^{-3 / 2} n^{-2}
$$

and when $q_{j}=2, j>1$, then the factor is

$$
c\left(\left(k_{j}-1-k_{j-1}\right) / n\right)^{-1 / 2} n^{-3 / 2} .
$$


For $j=1$, the factor is $n^{-1}$ or $n^{-1 / 2}$, depending on whether $q_{j}=2$ or 1 . Summing as in (43) shows that each factor corresponding to $\left(\Delta_{k_{j}}^{ \pm} Z^{2}\right)^{q_{j}}$ contributes $\mathrm{cn}{ }^{-1 / 2}$ to the sum of expectations provided $j>1$. The contribution from the first factor is either $c$ or $c n^{1 / 2}$. Hence

$$
\left|E\left(\sum_{k_{1}, \ldots, k_{m} \in \Theta}\left(\Delta_{k_{1}}^{ \pm} Z^{2}\right)^{q_{1}}\left(\Delta_{k_{2}}^{ \pm} Z^{2}\right)^{q_{2}} \ldots\left(\Delta_{k_{m}}^{ \pm} Z^{2}\right)^{q_{m}}\right)\right| \leq c n^{(-m+2) / 2} .
$$

If $m>3$ then

$$
\lim _{n \rightarrow \infty}\left|E\left(\sum_{k_{1}, \ldots, k_{m} \in \Theta}\left(\Delta_{k_{1}}^{ \pm} Z^{2}\right)^{q_{1}}\left(\Delta_{k_{2}}^{ \pm} Z^{2}\right)^{q_{2}} \ldots\left(\Delta_{k_{m}}^{ \pm} Z^{2}\right)^{q_{m}}\right)\right|=0
$$

Suppose that some $q_{j}$ 's are greater than 2 and at least one of them is odd (we need this assumption to prove (29)). The only part of the proof that will be affected by this change in the assumptions is that the powers of $r_{j}$ 's in (33) will increase. If every $q_{j}$ is equal to $2 p_{j}+1$ or $2 p_{j}+2$ for some integer $p_{j} \geq 0$ then instead of (44) we will have

$$
\left|E\left(\sum_{k_{1}, \ldots, k_{m} \in \Theta}\left(\Delta_{k_{1}}^{ \pm} Z^{2}\right)^{q_{1}}\left(\Delta_{k_{2}}^{ \pm} Z^{2}\right)^{q_{2}} \ldots\left(\Delta_{k_{m}}^{ \pm} Z^{2}\right)^{q_{m}}\right)\right| \leq c n^{-p_{1}-\cdots-p_{m}+(-m+2) / 2} .
$$

Next we will analyse the fourth moment of $V_{n}$ 's. We have

$$
\begin{aligned}
\left(\sum_{k \in \Theta} \Delta_{k}^{ \pm} Z^{2}\right)^{4}= & \sum_{\substack{j, k \in \Theta \\
j, k \text { distinct }}}\left(\Delta_{j}^{ \pm} Z^{2}\right)^{2}\left(\Delta_{k}^{ \pm} Z^{2}\right)^{2}+\sum_{k \in \Theta}\left(\Delta_{k}^{ \pm} Z^{2}\right)^{4} \\
& +c \sum_{\substack{j, k \in \Theta \\
j \neq k}}\left(\Delta_{j}^{ \pm} Z^{2}\right)^{3} \Delta_{k}^{ \pm} Z^{2}+c \sum_{\substack{j, k, m \in \Theta \\
m \neq j \neq k<m}}\left(\Delta_{j}^{ \pm} Z^{2}\right)^{2} \Delta_{k}^{ \pm} Z^{2} \Delta_{m}^{ \pm} Z^{2} \\
& +c \sum_{\substack{i, j, k, m \in \Theta \\
i<j<k<m}} \Delta_{i}^{ \pm} Z^{2} \Delta_{j}^{ \pm} Z^{2} \Delta_{k}^{ \pm} Z^{2} \Delta_{m}^{ \pm} Z^{2}
\end{aligned}
$$

It follows from $(26),(27),(45)$ and $(46)$ that

$$
\begin{gathered}
\lim _{n \rightarrow \infty} E\left(3 \sum_{\substack{j, k \in \Theta \\
j, k \text { distinct }}}\left(\Delta_{j}^{ \pm} Z^{2}\right)^{2}\left(\Delta_{k}^{ \pm} Z^{2}\right)^{2}\right)=27\left(s_{2}-s_{1}\right)^{2}, \\
\lim _{n \rightarrow \infty}\left|E\left(\sum_{k \in \Theta}\left(\Delta_{k}^{ \pm} Z^{2}\right)^{4}\right)\right|=0, \\
\lim _{n \rightarrow \infty} \mid E\left(c \sum_{\substack{j, k \in \Theta \\
j \neq k}}\left(\Delta_{j}^{ \pm} Z^{2}\right)^{3} \Delta_{k}^{ \pm} Z^{2}+c \sum_{\substack{j, k, m \in \Theta \\
m \neq j \neq k<m}}\left(\Delta_{j}^{ \pm} Z^{2}\right)^{2} \Delta_{k}^{ \pm} Z^{2} \Delta_{m}^{ \pm} Z^{2}\right. \\
\left.+c \sum_{\substack{i, j, k, m \in \Theta \\
i<j<k<m}} \Delta_{i}^{ \pm} Z^{2} \Delta_{j}^{ \pm} Z^{2} \Delta_{k}^{ \pm} Z^{2} \Delta_{m}^{ \pm} Z^{2}\right) \mid=0 .
\end{gathered}
$$


Hence

$$
\lim _{n \rightarrow \infty} E\left(\sum_{k \in \Theta} \Delta_{k}^{ \pm} Z^{2}\right)^{4}=27\left(s_{2}-s_{1}\right)^{2}
$$

More generally, suppose that $m>1$ is an integer. Then

$$
\begin{aligned}
\left(\sum_{k \in \Theta} \Delta_{k}^{ \pm} Z^{2}\right)^{2 m}=(2 m-1) ! ! & \sum_{\substack{k_{1}, \ldots, k_{m} \in \Theta \\
k_{1}, \ldots, k_{m} \text { distinct }}}\left(\Delta_{k_{1}}^{ \pm} Z^{2}\right)^{2}\left(\Delta_{k_{2}}^{ \pm} Z^{2}\right)^{2} \ldots\left(\Delta_{k_{m}}^{ \pm} Z^{2}\right)^{2} \\
& +\sum_{\substack{2\left(q_{1}+\cdots+q_{j}\right)=2 m \\
q_{1} \cdot \ldots \cdot q_{j} \geq 2}} c\left(q_{1}, \ldots, q_{j}\right) \sum_{\substack{k_{1}, \ldots, k_{m} \in \Theta \\
n_{k_{1}}}}\left(\Delta^{ \pm} Z^{2}\right)^{2 q_{1}}\left(\Delta_{k_{2}}^{ \pm} Z^{2}\right)^{2 q_{2}} \ldots\left(\Delta_{k_{j}}^{ \pm} Z^{2}\right)^{2 q_{j}} \\
& +\sum_{q_{1}+\cdots+q_{j}=2 m} c\left(q_{1}, \ldots, q_{j}\right) \sum_{k_{1}, \ldots, k_{j} \in \Theta}\left(\Delta_{k_{1}}^{ \pm} Z^{2}\right)^{q_{1}}\left(\Delta_{k_{2}}^{ \pm} Z^{2}\right)^{q_{2}} \ldots\left(\Delta_{k_{j}}^{ \pm} Z^{2}\right)^{q_{j}}
\end{aligned}
$$

where $q_{i}$ 's are positive integers and in the last sum, at least two $q_{i}$ 's are odd. By (27)

$$
\lim _{n \rightarrow \infty} E\left((2 m-1) ! ! \sum_{\substack{k_{1}, \ldots, k_{m} \in \Theta \\ k_{1}, \ldots, k_{m} \text { distinct } \\ 2 m=q}}\left(\Delta_{k_{1}}^{ \pm} Z^{2}\right)^{2}\left(\Delta_{k_{2}}^{ \pm} Z^{2}\right)^{2} \ldots\left(\Delta_{k_{m}}^{ \pm} Z^{2}\right)^{2}\right)=(2 m-1) ! ! 3^{m}\left(s_{2}-s_{1}\right)^{m}
$$

We have

$$
\begin{aligned}
\lim _{n \rightarrow \infty} E\left(\sum_{\substack{2\left(q_{1}+\cdots+q_{j}\right)=2 m \\
q_{1} \cdot \ldots \cdot q_{j} \geq 2}} c\left(q_{1}, \ldots, q_{j}\right) \sum_{k_{1}, \ldots, k_{m} \in \Theta}\left(\Delta_{k_{1}}^{ \pm} Z^{2}\right)^{2 q_{1}}\left(\Delta_{k_{2}}^{ \pm} Z^{2}\right)^{2 q_{2}} \ldots\left(\Delta_{k_{j}}^{ \pm} Z^{2}\right)^{2 q_{j}}\right. \\
\left.\quad+\sum_{q_{1}+\cdots+q_{j}=2 m} c\left(q_{1}, \ldots, q_{j}\right) \sum_{k_{1}, \ldots, k_{j} \in \Theta}\left(\Delta_{k_{1}}^{ \pm} Z^{2}\right)^{q_{1}}\left(\Delta_{k_{2}}^{ \pm} Z^{2}\right)^{q_{2}} \ldots\left(\Delta_{k_{j}}^{ \pm} Z^{2}\right)^{q_{j}}\right)=0
\end{aligned}
$$

by (26), (45) and (46). We conclude that

$$
\lim _{n \rightarrow \infty} E\left(\sum_{k \in \Theta} \Delta_{k}^{ \pm} Z^{2}\right)^{2 m}=(2 m-1) ! ! 3^{m}\left(s_{2}-s_{1}\right)^{m}
$$

for $m>1$. Note that

$$
\lim _{n \rightarrow \infty} E\left(\sum_{k \in \Theta} \Delta_{k}^{ \pm} Z^{2}\right)^{2 m+1}=\lim _{n \rightarrow \infty} 0=0
$$

since the random variables under the expectation have symmetric distributions.

We see from (49)-(50) that the moments of $\widetilde{V}_{n}\left(s_{2}\right)-\widetilde{V}_{n}\left(s_{1}\right) \stackrel{\text { df }}{=} \sum_{k \in \Theta} \Delta_{k}^{ \pm} Z^{2}$ (except possibly the second moment) converge as $n$ goes to infinity to the moments of the normal distribution with mean 0 and variance $3\left(s_{2}-s_{1}\right)$. The difference

$$
\left|\widetilde{V}_{n}\left(s_{2}\right)-\widetilde{V}_{n}\left(s_{1}\right)-\left(V_{n}\left(s_{2}\right)-V_{n}\left(s_{1}\right)\right)\right|
$$


is bounded by $\left|\Delta_{j}^{ \pm} Z^{2}+\Delta_{k}^{ \pm} Z^{2}\right|$ for appropriate $j$ and $k$. Since

$$
\lim _{n \rightarrow \infty} E\left|\Delta_{j}^{ \pm} Z^{2}+\Delta_{k}^{ \pm} Z^{2}\right|^{p}=0
$$

for every $p<\infty$ and every choice of $j$ and $k$, an application of Minkowski's inequality shows that the moments of $V_{n}\left(s_{2}\right)-V_{n}\left(s_{1}\right)$ have the same limits as those of $\widetilde{V}_{n}\left(s_{2}\right)-\widetilde{V}_{n}\left(s_{1}\right)$.

It is perhaps appropriate to explain why we have not proved the convergence of the second moments of $V_{n}\left(s_{2}\right)-V_{n}\left(s_{1}\right)$. In order to do it we would have to have very accurate estimates of

$$
E\left(\sum_{j, k \in \Theta} \Delta_{j}^{ \pm} Z^{2} \Delta_{k}^{ \pm} Z^{2}\right)
$$

which cannot be found using our method.

A simple modification of the proof of Theorem 4.5.5 of Chung (1974) shows that every subsequence of $\left\{V_{n}\left(s_{2}\right)-V_{n}\left(s_{1}\right)\right\}_{n>1}$ has a further subsequence which converges in distribution to a random variable which has the same moments (with possible exception of variance) as the centered normal with variance $3\left(s_{2}-s_{1}\right)$. Lemma 1 implies that there is only one distribution which has the same moments of order greater than 2 as $N\left(0,3\left(s_{2}-s_{1}\right)\right)$ and so $\left\{V_{n}\left(s_{2}\right)-V_{n}\left(s_{1}\right)\right\}_{n \geq 1}$ converges in distribution to $N\left(0,3\left(s_{2}-s_{1}\right)\right)$.

We will indicate how one can prove that the finite-dimensional distributions of $V_{n}$ also converge to those of Brownian motion $B(s)$ with variance $3 s$. In order to prove that a pair of random variables has a two-dimensional normal distribution it suffices to show that all linear combinations of the random variables are normal. One can show this by finding the moments of all linear combinations. Let us fix some $0 \leq s_{1}<s_{2} \leq u_{1}<u_{2}$ and let $\Theta(s)$ and $\Theta(u)$ be the obvious analogues of $\Theta$. Let $a$ and $b$ be arbitrary real numbers. In order to find

$$
\lim _{n \rightarrow \infty} E\left(a \sum_{k \in \Theta(s)} \Delta_{k}^{ \pm} Z^{2}+b \sum_{k \in \Theta(u)} \Delta_{k}^{ \pm} Z^{2}\right)^{m}
$$

we might calculate

$$
\lim _{n \rightarrow \infty} E\left[\left(a \sum_{k \in \Theta(s)} \Delta_{k}^{ \pm} Z^{2}\right)^{m_{1}}\left(b \sum_{k \in \Theta(u)} \Delta_{k}^{ \pm} Z^{2}\right)^{m_{2}}\right]
$$

for various values of $m_{1}$ and $m_{2}$. Doing this would require estimates of

$$
\begin{aligned}
E\left(\sum_{k_{1}, \ldots, k_{m} \in \Theta(s)}\left(\Delta_{k_{1}}^{ \pm} Z^{2}\right)^{q_{1}}\left(\Delta_{k_{2}}^{ \pm} Z^{2}\right)^{q_{2}} \ldots\left(\Delta_{k_{m}}^{ \pm} Z^{2}\right)^{q_{m}} \times\right. & \\
& \left.\times \sum_{k_{1}, \ldots, k_{i} \in \Theta(u)}\left(\Delta_{k_{1}}^{ \pm} Z^{2}\right)^{p_{1}}\left(\Delta_{k_{2}}^{ \pm} Z^{2}\right)^{p_{2}} \ldots\left(\Delta_{k_{i}}^{ \pm} Z^{2}\right)^{p_{i}}\right) .
\end{aligned}
$$

It can be shown that these expectations converge to the desired limits. The method of proof is a routine adaptation of the one used in the case of the one-dimensional distributions of $V_{n}$. We omit the details as they are tedious. The case of $m$-dimensional distributions, $m>2$, can be dealt with in the same way.

Since the the finite-dimensional distributions of $V_{n}$ converge as $n \rightarrow \infty$ to those of $B$ it remains to check that the distributions of $V_{n}$ are tight. It will suffice to show that there exists $c<\infty$ such that

$$
E\left(V_{n}\left(s_{2}\right)-V_{n}\left(s_{1}\right)\right)^{4} \leq c\left(s_{2}-s_{1}\right)^{2}
$$


for all $s_{1}, s_{2}$ and all $n$ (see Billingsley (1968), (12.51)). It follows easily from the scaling properties of Brownian motions $X$ and $Y$ that $Z$ has the "4-th order" scaling properties while $V_{n}$ 's have the Brownian scaling properties. In other words, the distribution of $\left\{\sqrt{n / m} V_{n}(s \cdot m / n), s \geq 0\right\}$ is the same as that of $\left\{V_{m}(s), s \geq 0\right\}$. It follows that (51) holds for all $n$ if it holds for a single $n$. We will show that (51) holds for $n=1$. Suppose that $s_{1}=0$. Then we want to show that

$$
E\left(V_{1}\left(s_{2}\right)\right)^{4} \leq c s_{2}^{2}
$$

This is true for $s_{2}=1$ and some $c$ since $V_{1}$ has all moments. It is easy to extend it to all $s_{2} \in[0,1]$ since $V_{1}$ is (by definition) a linear function on $[0,1]$. Suppose that $s_{2}$ is an integer greater than 1 . Then

$$
E\left(V_{1}\left(s_{2}\right)\right)^{4}=E\left(\sqrt{s_{2}} V_{s_{2}}\left(s_{2} / s_{2}\right)\right)^{4}=s_{2}^{2} E\left(V_{s_{2}}(1)\right)^{4}
$$

by the scaling property of $V_{n}$ 's. We have proved in (48) that

$$
\limsup _{s_{2} \rightarrow \infty} E\left(V_{s_{2}}(1)\right)^{4}<c<\infty
$$

SO

$$
E\left(V_{1}\left(s_{2}\right)\right)^{4} \leq c s_{2}^{2}
$$

for all integer $s_{2}$. It is elementary to extend the inequality to non-integer $s_{2}$ using the fact that $V_{1}$ is a linear function between integers. Now we use another invariance property of $V_{n}$ 's, namely, that of translation invariance of their increments. The distribution of $\left\{V_{1}(s)-V_{1}(0), s \geq 0\right\}$ is the same as that of $\left\{V_{1}(s+a)-V_{1}(a), s \geq 0\right\}$ provided $a>0$ is an integer. This and (52) imply that

$$
E\left(V_{1}\left(s_{2}\right)-V_{1}\left(s_{1}\right)\right)^{4} \leq c\left(s_{2}-s_{1}\right)^{2}
$$

for integer numbers $s_{1}$ and all $s_{2}>s_{1}$. Extending the result to all $s_{1}$ is easy.

This concludes the proof of the weak convergence of $V_{n}$ 's to $B$.

Proof of Theorem 1 (ii). We will prove the theorem only for $s=0$ and $t=1$. The estimate (46) obtained in the proof of Theorem 2 will be used to prove (4). We will apply the estimate with $s_{1}=0$ and $s_{2}=t$ (recall the notation form the previous proof). We want to show that

$$
\lim _{n \rightarrow \infty} E\left(\sum_{j=1}^{n}\left(\Delta_{j} Z\right)^{3}\right)^{p}=0
$$

It will suffice to show that

$$
\lim _{n \rightarrow \infty}\left|E\left(\sum_{k_{1}, \ldots, k_{m} \in \Theta}\left(\Delta_{k_{1}} Z\right)^{3 q_{1}}\left(\Delta_{k_{2}} Z\right)^{3 q_{2}} \ldots\left(\Delta_{k_{m}} Z\right)^{3 q_{m}}\right)\right|=0
$$

for integer $q_{j} \geq 1$ such that $q_{1}+\cdots+q_{m}=p$. The sum extends over distinct $k_{j}$ 's. If all $q_{j}$ 's are even then (53) follows from (26).

Assume that at least one of $q_{j}$ 's is odd. Let us adopt the following convention for integer $m$

$$
\left(\Delta_{k}^{ \pm} Z^{2}\right)^{m+1 / 2}=\left(\Delta_{k} Z\right)^{2 m+1} .
$$


One can verify that (46) remains true if some of $q_{j}$ 's are not integers but have the form $k+1 / 2$ for some integer $k$. Such $q_{j}$ 's should be treated as odd integers for the purpose of the decomposition $q_{j}=2 p_{j}+1$ used in (46). We obtain from (46)

$$
\left|E\left(\sum_{k_{1}, \ldots, k_{m} \in \Theta}\left(\Delta_{k_{1}} Z\right)^{3 q_{1}}\left(\Delta_{k_{2}} Z\right)^{3 q_{2}} \ldots\left(\Delta_{k_{m}} Z\right)^{3 q_{m}}\right)\right| \leq c n^{-r_{1}-\cdots-r_{m}+(-m+2) / 2}
$$

where $3 q_{j} / 2=2 r_{j}+2$ if $3 q_{j} / 2$ is an even integer and $3 q_{j} / 2=2 r_{j}+1$ otherwise. If $m>2$ then (54) implies (53). If $m=2$ and $p$ is large then $r_{1}+r_{2}>0$ and again (54) implies (53).

\section{REFERENCES}

1. P. Billingsley, Convergence of Probability Measures, Wiley, New York, 1968.

2. K. Burdzy, Some path properties of iterated Brownian motion, Seminar on Stochastic Processes 1992 (E. Çinlar, K.L. Chung and M. Sharpe, eds.), Birkhäuser, Boston, 1993, pp. 67-87.

3. R. Durrett, Probability: Theory and Examples, Wadsworth, Belmont, CA, 1991.

4. T. Funaki, Probabilistic construction of the solution of some higher order parabolic differential equations, Proc. Japan Acad. 55 (1979), 176-179.

5. K.J. Hochberg, A signed measure on path space related to Wiener measure, Ann. Probab. 6 (1978), 433-458.

6. V. Yu. Krylov, Some properties of the distribution corresponding to the equation $\partial u / \partial t=(-1)^{q+1} \partial^{2 q} u / \partial x^{2 q}$, Soviet Math. Dokl. 1 (1960), 760-763.

7. A. Mạdrecki, The 4-a-stable motions, construction, properties and applications, (preprint) (1992).

8. A. Mądrecki and M. Rybaczuk, New Feynman-Kac type formula, Reports Math. Phys (1992), (to appear).

Department of Mathematics, GN-50, Seattle, WA 98195 